\title{
Monte Carlo Calculations of Gamma-ray Spectra for Calibration
}

\author{
Tzu-Fang Wang
}

December 1996

This is an informal report intended primarily for internal or limited external distribution. The opinions and conclusions stated are those of the author and may or may not be those of the Laboratory. 


\section{DISCLAIMER}

This document was prepared as an account of work sponsored by an agency of the United States Government. Neither the United States Government nor the University of California nor any of their employees, makes any warranty, express or implied, or assumes any legal liability or responsibility for the accuracy, completeness, or usefulness of any information, apparatus, product, or process disclosed, or represents that its use would not infiringe privately owned rights. Reference herein to anj specific commercial products, process, or service by trade name, trademark, manufacturer, or otherwise, does not necessarily constitute or imply its endorsement, recommendation, or favoring by the United States Government or the University of California. The views and opinions of authors expressed herein do not necessarily state or reflect those of the United States Government or the University of California, and shall not be used for advertising or product endorsement purposes.

This report has been reproduced

direetly from the best avallable cops.

Available to DOE and DOE contractors from the Omice of Scientific and Technical Information

P.O. Box 62, Oak Ridge, TN 37831

Prices available from (615) 576-8401, FTS 626-8401

Available to the public from the

National Tectuical Information Service

US. Department of Commerce

5285 Port Royal Rd.

Springfield, VA 22161 


\title{
Monte Carlo Calculations of Gamma-ray Spectra for Calibration
}

\author{
Tzu-Fang Wang \\ Lawrence Livermore National Laboratory \\ Livermore, California 94550
}

\begin{abstract}
We have developed a methodology to simulate gamma-ray energy spectra to aid those who assay special nuclear materials (SNM) such as plutonium or uranium. For example, when SNM are moved from one facility to the another, the amount of SNM moved is subtracted from the former's inventory and added to the inventory of the latter. To ensure that identical amounts of SNM are shipped and received, the shipper performs measurements prior to shipping, and the receiver performs measurements after receipt.
\end{abstract}

Shippers and receivers often determine the amount of SNM in a package with the aid of an energy histogram, or spectrum, of gamma-rays emitted from the SNM using a germanium detector. The gamma-ray energies and intensities recorded in the spectrum are then analyzed by computer to accurately determine the isotopic content of SNM. The performance of the detection system and the isotopic analysis software is calibrated using physical isotopic standards to assure accuracy.

Unfortunately, the isotopic standards of SNM are difficult to make and certify, and therefore, costly. However, computer simulation can be a cost-effective, timely, and convenient tool to create gamma-ray spectra representative of isotopic standards. These simulations, while not substituting completely for physical standards, can help test and qualify isotopic analysis software; investigate questions of absorbers, collimation, and measurement geometry; study the effects of various mixtures of radioactive species on the assay; investigate the effects of nonuniform materials; and simulate the effects of electronic or detector degradation.

The gamma-rays emitted from the standard sources, the gamma-ray interactions with the detectors, the effects of electronics components, and the influence of the geometry in the measurement setup can all be simulated since the detailed physics of all the processes are known. The Monte Carlo method, a "dice-throwing" sampling scheme, is used in computer simulations because of the probabilistic nature of the interactions involved.

We have developed a detailed simulation methodology that has four main components: one each for the source, the detection geometry and gamma-ray interactions, the electronics, and our isotopic analysis software. Figure 1 shows the first three simulation components and the corresponding modules in experimental measurement. The source component is a data base of gamma ray data for SNM isotopes, and a computer program 
GAMGEN to calculate the radioactive decay of the isotopes developed at the Lawrence Livemore National Laboratory (LLNL). The geometry and gamma ray interactions component consists of a user interface to create the input data for the Monte Carlo program, MCNP, the widely used Monte Carlo interaction program created by the Los Alamos National Laboratory. The effects of the electronic and detector response are simulated by a post-processing program POSTGL developed at LLNL. Finally, SNM energy spectra, both simulated and measured, can be analyzed by Multi-Group Analysis (MGA), an internationally used software package developed at LLNL.

We have demonstrated that our methodology can successfully simulate plutonium gamma-ray standards with the ${ }^{239} \mathrm{Pu}$ enrichment ranging from $60 \%$ to $90 \%$. For example, for one of our simulated weapons-grade plutonium gamma-ray standards, MGA reported identical (within statistical errors) isotopic contents for the measured gamma-ray spectrum and the computer simulation of that spectrum, shown in Figure 2.

We are extending our capability to simulate the gamma-ray spectra of uranium isotopic standards. With completion of this effort, we can easily extend the methodology to calculate the spectra of mixed plutonium and uranium standards. Physical standards of such mixtures can be difficult to make due to the difference in the density between uranium and plutonium, and the desire to refrain from mixing SNM elements if possible. Samples of such mixed SNM do exist in United States' inventories, however, simulation of their gamma-ray spectra can be an aid to analysts in choosing the correct software or measurement scheme.

Using the methodology we have developed, anyone can install our simulation capability on his or her personal computer. A modern personal computer can provide a simulated spectrum sufficient to address questions such a the effect of a particular collimator or absorber in a few hours. A simulation calculated over a weekend can provide a gamma- ray spectrum that is almost impossible to distinguish from the measured spectrum of a physical standard.

This work was performed under the auspices of the U.S. DOE by the LLNL under Contract No. W-7405-ENG-48. Work was supported by the Office of Safeguards and Security.

\section{Figure Captions:}

Figure 1. Three of the computer software components of our methodology for calculating gamma-ray spectra of SNM are identified with the physical process and components they simulate.

Figure 2. A comparison of a measured spectrum of a plutonium standard with the simulation of that standard spectrum. The MGA fits to those spectra are shown. The measured and simulated spectra give identical isotopes assays. 


\section{GAMGEN}
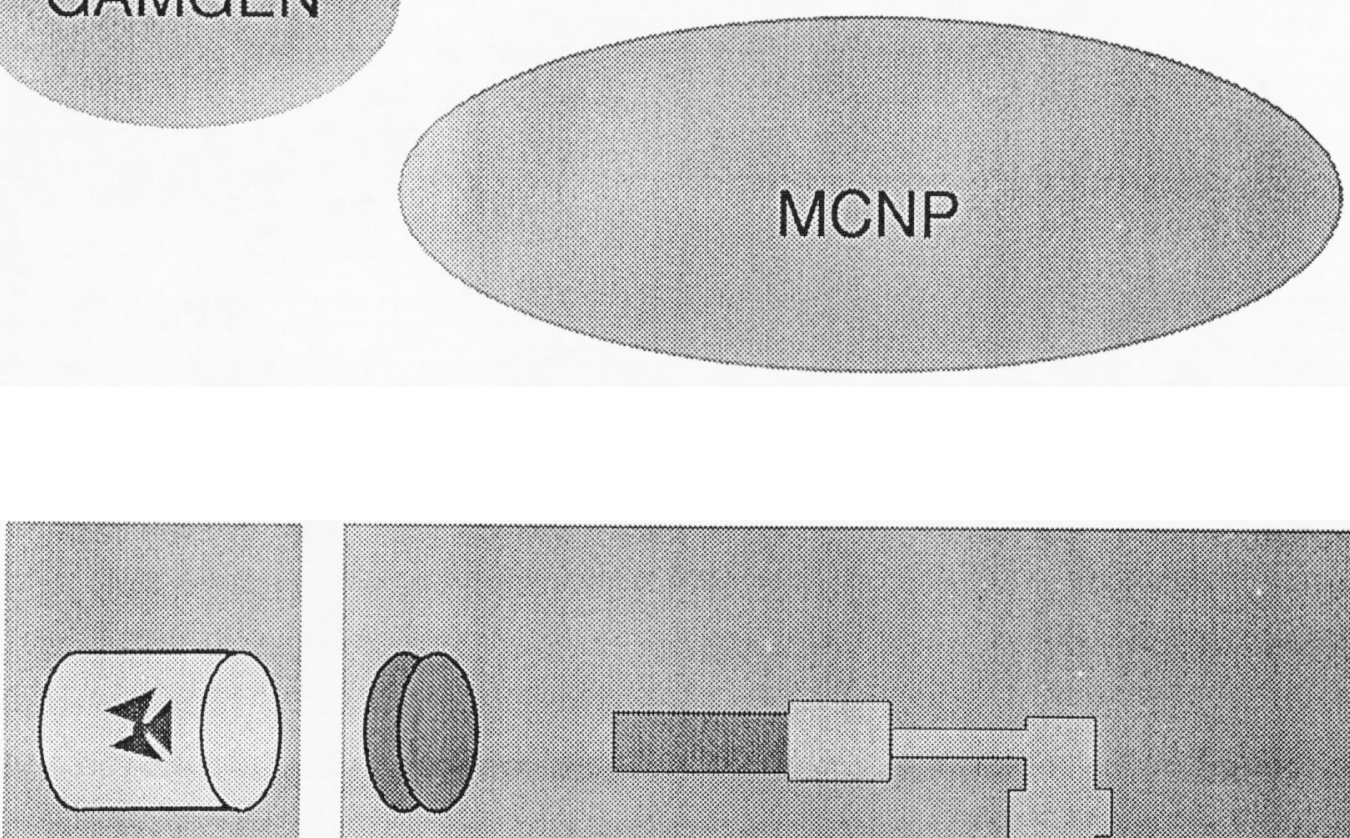

source
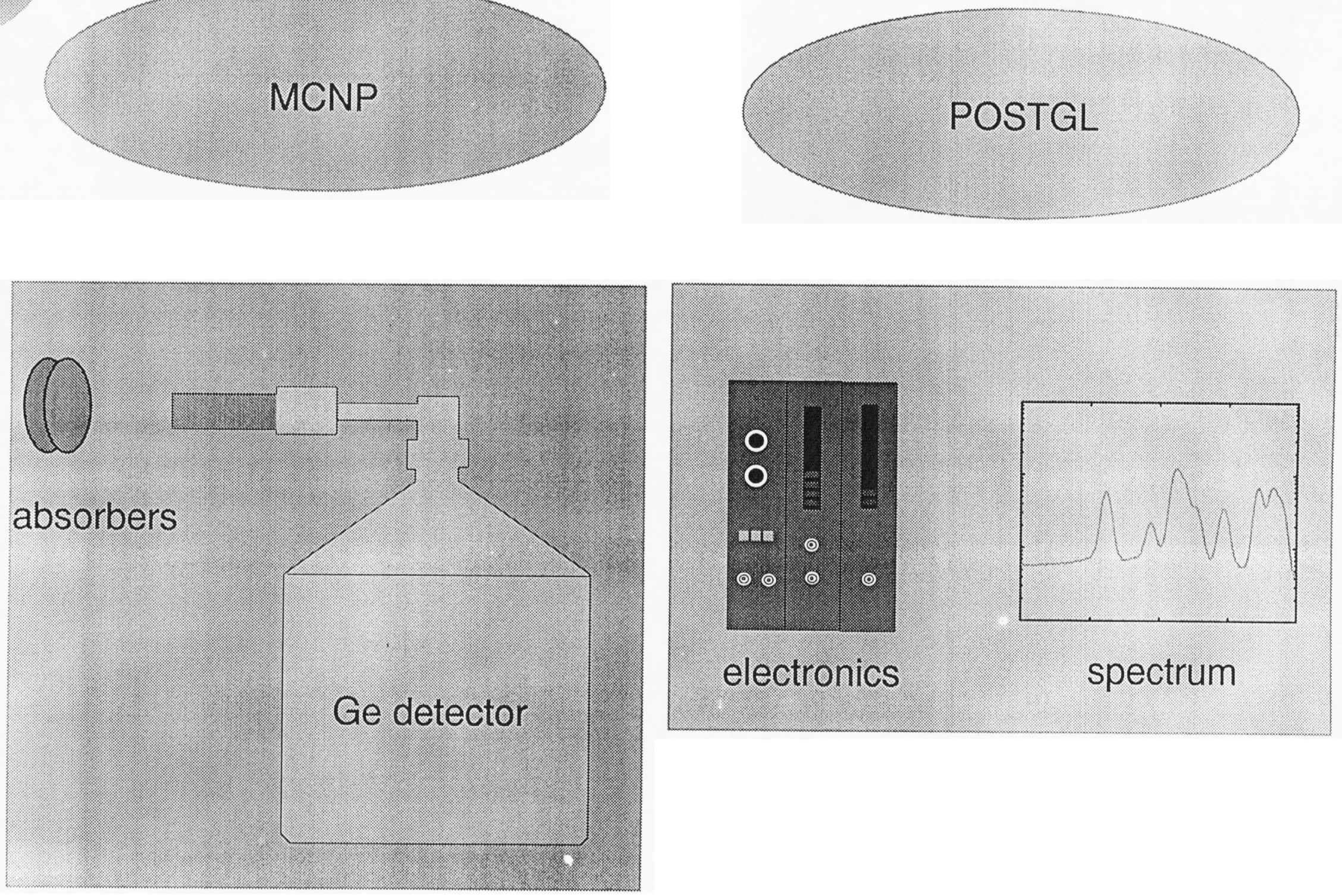

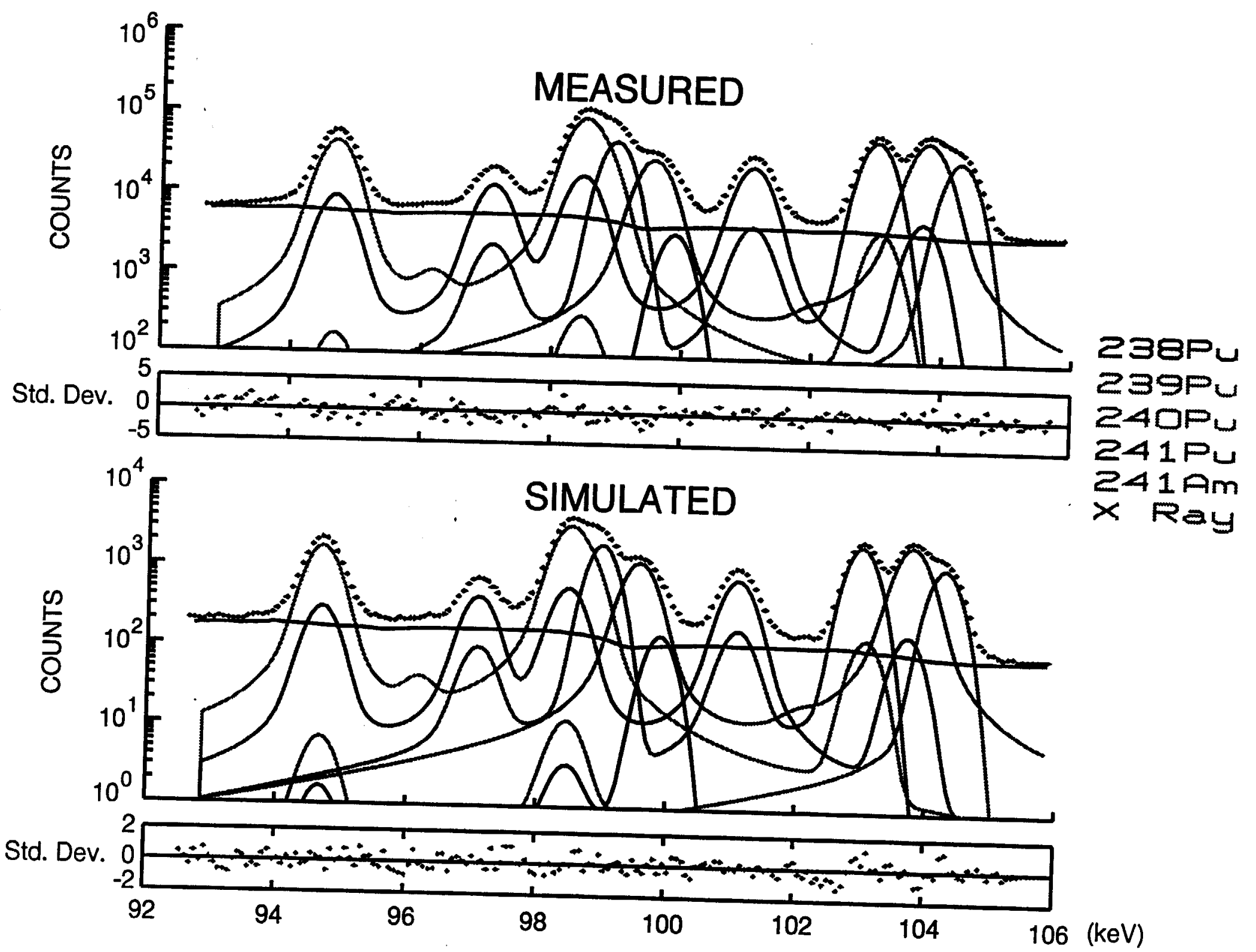


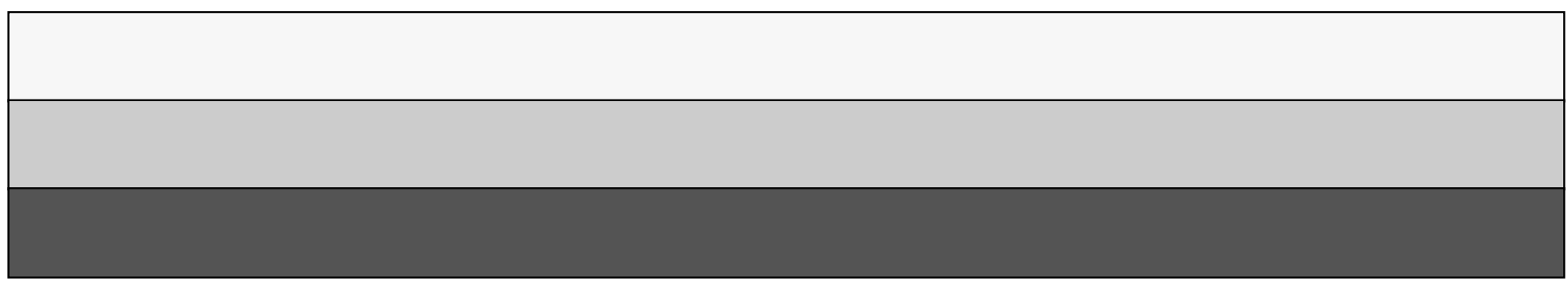

